\title{
PROMOCIÓN DE LA LECTURA EN LAS BIBLIOTECAS PÚBLICAS DE CATALUÑA: EVALUACIÓN Y RESULTADOS
}

Mònica Baró, Teresa Mañà, Maite Barrios y Júlia Baena
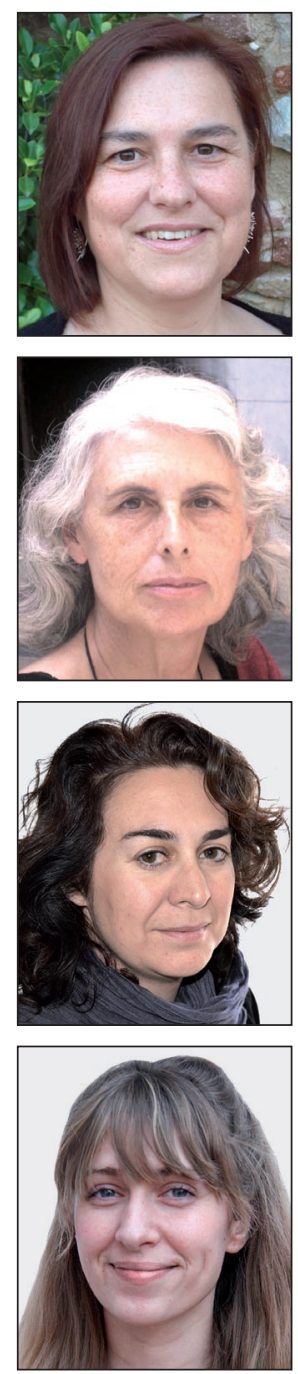

Mònica Baró es profesora titular de la Facultat de Biblioteconomia i Documentació de la Universitat de Barcelona (UB). Licenciada en historia, diplomada en biblioteconomía y doctora en documentación. Miembro del Observatori de Biblioteques, Llibres i Lectura de la UB desde su creación en 2009.

Universitat de Barcelona Facultat de Biblioteconomia i Documentació Melcior de Palau, 140. 08014 Barcelona baro@ub.edu

Teresa Mañà es profesora titular de la Facultat de Biblioteconomia i Documentació de la Universitat de Barcelona (UB). Licenciada en filología, diplomada en biblioteconomía y doctora en documentación. Ha sido directora del Observatori de Biblioteques, Llibres i Lectura de la UB desde su creación en 2009.

mana@ub.edu

Maite Barrios es profesora agregada del Departamento de Metodología de las Ciencias del Comportamiento de la Universitat de Barcelona (UB). Licenciada y doctora en psicología. Desarrolla su actividad docente e investigadora en la Facultad de Biblioteconomía y Documentación de la UB desde 2002. Es miembro del Observatori de Biblioteques, Llibres i Lectura de la UB desde 2009.

mbarrios@ub.edu

Júlia Baena es técnica superior en diseño gráfico y diplomada en biblioteconomía y documentación. Realiza tareas de apoyo a la investigación del Observatori de Biblioteques, Llibres i Lectura y del grupo de investigación Bescolar. Colabora en tareas de documentación con el Departament d'Ensenyament (Generalitat de Catalunya) i el Col/legi Oficial de Bibliotecaris-Documentalistes de Catalunya.

julietabaena@gmail.com

\section{Resumen}

Se presentan los resultados de una encuesta realizada en 2009 entre las 320 bibliotecas públicas de Cataluña sobre las prácticas de evaluación de las acciones de promoción de la lectura. Los resultados se basan tanto en los datos cuantitativos como en las opiniones y las valoraciones del personal directivo de las bibliotecas en torno a estas actividades.

\section{Palabras clave}

Bibliotecas públicas, Evaluación, Promoción de la lectura.

\section{Title: Reading promotion in public libraries in Catalonia: evaluation and results}

\section{Abstract}

Results of a 2009 survey of the 320 public libraries in Catalonia that explored the practices of evaluating actions taken to promote reading. The results are based on both quantitative data and the opinions and assessments of library administrators concerning these activities. 


\section{Keywords}

Public libraries, Evaluation, Reading promotion.

\section{Introducción}

En un contexto de crisis en el que lo que cuenta son los resultados, cabe preguntarse sobre la evaluación de las acciones de fomento de la lectura en las bibliotecas públicas: ¿se evalúan? ¿cómo? ¿con qué finalidad?

El artículo trata de dar respuesta a las prácticas relativas a la evaluación de las acciones para la promoción de la lectura en las bibliotecas públicas de Cataluña a partir de las opiniones y valoraciones de los profesionales que las dirigen. Dichas prácticas se identificaron en el contexto de un estudio más amplio (Baró; Mañà, 2009) propuesto por la Subdirección General de Bibliotecas de la Generalitat de Catalunya al Observatori de Biblioteques, Llibres i Lectura de la Facultat de Biblioteconomia i Documentació de la Universitat de Barcelona, con el doble objetivo de 1) valorar los procesos de planificación, seguimiento y evaluación de la calidad de las actividades de promoción de la lectura llevadas a cabo por las bibliotecas públicas catalanas y 2) definir indicadores e instrumentos para que los centros pudieran valorar el impacto de estas acciones, establecer los procesos de evaluación y modificar sus propuestas en función de los resultados (Baró et al., 2010). Para realizar este estudio, se visitaron 21 bibliotecas y se entrevistaron bibliotecarios, usuarios y profesionales que trabajan en el ámbito de la promoción de la lectura. Además, se distribuyó un cuestionario online a todas las bibliotecas del sistema para obtener datos significativos, tanto cuantitativos como cualitativos, parte de cuyos resultados exponemos a continuación.

http://www.ub.edu/obll

Aun cuando en el entorno de la biblioteca pública la evaluación es una práctica cada vez más extendida que se aplica a distintos aspectos como la eficacia de los servicios, el uso de la colección y otros, la investigación y la literatura profesional específicas sobre los resultados de las acciones de promoción de la lectura son muy limitadas (Farmer; Stricevic, 2011), y suelen abordarse en un contexto más amplio de evaluación de servicios bibliotecarios en general. La bibliografía disponible proviene, esencialmente, de los países anglosajones y se relaciona con la evaluación de las acciones desarrolladas por las bibliotecas públicas en el contexto de los distintos planes gubernamentales de promoción de la lectura. En estos casos, algunos autores establecen la dificultad de evaluar la eficacia de dichas acciones en términos cualitativos tales como la modificación de hábitos lectores, incremento del nivel lector, etc. (Train; Elkin, 2001) y se centran en la evaluación de los procesos en sí mismos, valorando la eficacia de los métodos en relación con el cumplimiento de los objetivos estratégicos de dichos planes y de cada una de las acciones (Wilson; Train, 2004). En otros casos se evidencia la dificultad de contar con herramientas fiables (y contrastables) para la recopilación de datos y el establecimiento de indicadores (Greenwood; Davies, 2004). En nuestro caso, la revisión bibliográfica permitió comprobar la ausencia de estudios propios y centrados en estos aspectos y establecer los aspectos clave que articularían el cuestionario.

Éste se elaboró y se distribuyó mediante un software de encuestas online y constaba de 21 preguntas. La encuesta se dirigió a principios de 2009 al total de las 348 bibliotecas públicas catalanas, de las cuales respondieron 197 (56,6\%) en un plazo de dos meses. Este porcentaje de respuesta ofrece una elevada fiabilidad de los datos obtenidos.

Se han obtenido los indicadores descriptivos en función del tipo de variable (porcentaje o bien media y desviación típica) y se ha realizado una regresión logística para valorar el efecto de distintos factores sobre la práctica de evaluar todas o parte de las actividades de promoción de la lectura que se programan. Dichos factores son: actividades programadas por los servicios externos de apoyo a las bibliotecas, número de personas que trabajan en las bibliotecas, número total de actividades programadas, así como la interacción entre el número de personas que trabajan en las bibliotecas y el número de actividades programadas.

\section{Evaluación de la promoción de la lectura}

Como el conjunto de bibliotecas españolas, las bibliotecas públicas de Cataluña dedican gran parte de su tiempo y de sus energías en diseñar y desarrollar acciones de índole diversa que tienen como objetivo la promoción de la lectura. Según los datos estadísticos de la Subdirecció General de Biblioteques, en 2010 se realizaron 34.966 actividades en 348 bibliotecas públicas de Cataluña, lo que da una media de 100 actividades/centro. Sin embargo, según las respuestas de las 197 bibliotecas que contestaron a nuestra encuesta, la media de actividades fue de 75 por centro, lo que aun así supone más de una a la semana [mediana $=50$, desviación típica $=72,14]$. La variabilidad entre centros es muy elevada, tal como indica el valor de la desviación típica.

Aunque se observa una relación directa y moderada entre el número de personas que trabajan en una biblioteca y el número de actividades que se programan $(r=0,49 ; p<0,001)$, parece que su evaluación no tiene relación ninguna con el volumen de personal, ni con el número de actividades realizadas ni, tampoco, con el hecho de que éstas se programen a partir de ofertas externas a la biblioteca.

Sobre la eficacia de dichas prácticas, planean dudas bien fundamentadas, máxime cuando los estudios evidencian que las prácticas lectoras de los españoles se sitúan en los puestos de cola. Pero lo cierto es que aunque la mayoría 
de las bibliotecas públicas preguntadas considera necesario evaluar estas actividades (80,5\%), ésta no es todavía una práctica generalizada y tan sólo un $48,4 \%(n=93)$ de ellas manifiesta evaluar todas las actividades de promoción de la lectura que organizan, un $41,7 \%(n=80)$ evalúa tan sólo algunas de ellas y un $9,9 \%(n=19)$ no evalúa ninguna actividad.

El estudio muestra que no existen modelos ni indicadores específicos para evaluar las actividades de promoción de la lectura y que, en la mayoría de casos, suele recurrirse a la contabilización del público asistente como principal indicador de éxito $(97,3 \%)$, aunque también se considera como indicador el grado de satisfacción de los usuarios $(88,4 \%)$. Otros datos objetivos y cuantificables son utilizados por un número mucho menor de centros: el $38 \%$ contabiliza el número de préstamos de documentos relacionados con las actividades, y el 34\% tiene en cuenta el aporte de nuevos públicos a la biblioteca. Todavía son menos $(16,2 \%)$ los centros que computan el número de nuevos carnets realizados después de cada sesión.

En la figura 1 se muestran los indicadores que se utilizan para evaluar las actividades de promoción de la lectura y su frecuencia de uso.

Las bibliotecas procuran recabar otras informaciones cualitativas para determinar el éxito de dichas actividades. Así, para conocer la opinión de los usuarios, las conversaciones informales con los asistentes son el procedimiento más habitual. También se tienen en cuenta las valoraciones del personal de la biblioteca, pero no suelen obtenerse ni analizarse las valoraciones del conductor de las actividades realizadas, como se indica en la tabla 1.

La evaluación no se realiza de manera sistemática. Se preguntó sobre las causas que en opinión de los responsables de las bibliotecas obstaculizan dicha evaluación. En primer lugar se situaría la falta de instrumentos preparados, es-

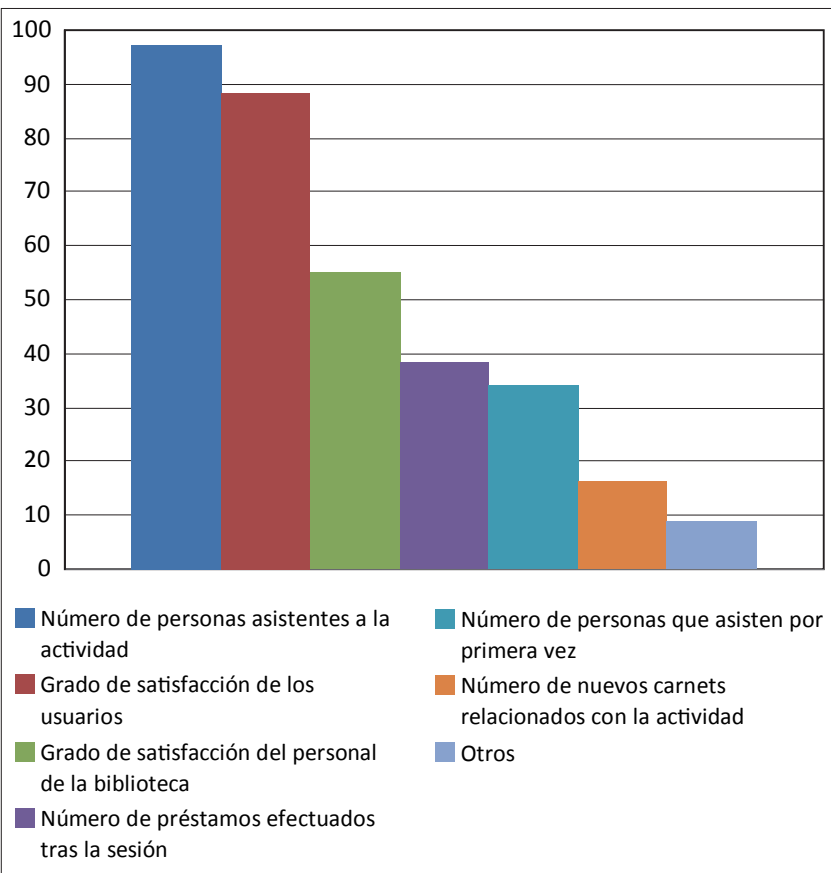

Figura 1. Frecuencia de uso de determinados indicadores para evaluar las actividades de promoción de la lectura

\begin{tabular}{|l|c|c|}
\hline & Media & DT \\
\hline Conversaciones informales con los usuarios & 6,06 & 1,06 \\
\hline Valoraciones del personal de la biblioteca & 5,87 & 1,38 \\
\hline $\begin{array}{l}\text { Cuestionarios de evaluación del conductor de } \\
\text { la actividad }\end{array}$ & 3,25 & 2,17 \\
\hline Cuestionarios de satisfacción del público & 3,03 & 1,97 \\
\hline Entrevistas formales con los usuarios & 2,64 & 1,99 \\
\hline
\end{tabular}

Escala de valoración de 1 a 7 puntos. A mayor puntuación, más de acuerdo con la afirmación (DT = desviación típica)

Tabla 1. Aplicación de distintos métodos para evaluar las actividades de promoción de la lectura

tandarizados y eficaces. El hecho de que los usuarios se muestren reacios a responder encuestas y cuestionarios es visto también como un impedimento aunque en menor medida. La dificultad de establecer relaciones causa-efecto entre las actividades y la promoción efectiva de la lectura es la razón aducida en algunos casos puesto que resulta difícil medir su eficacia en este sentido. Es destacable que algunos encuestados manifiestan claramente que no es necesario evaluar dichas actividades. Los resultados pueden verse en la tabla 2.

\begin{tabular}{|l|c|c|}
\hline & Media & DT \\
\hline $\begin{array}{l}\text { No se dispone de instrumentos eficaces para } \\
\text { la evaluación }\end{array}$ & 4,32 & 1,93 \\
\hline $\begin{array}{l}\text { Los usuarios son reacios a responder encuestas } \\
\text { y cuestionarios }\end{array}$ & 3,94 & 1,92 \\
\hline $\begin{array}{l}\text { No se puede establecer una relación directa } \\
\text { entre las actividades y la promoción real de la } \\
\text { lectura }\end{array}$ & 3,53 & 1,80 \\
\hline $\begin{array}{l}\text { No es necesario evaluar este tipo de activida- } \\
\text { des }\end{array}$ & 2,24 & 1,62 \\
\hline
\end{tabular}

Escala de valoración de 1 a 7 puntos. A mayor puntuación, más de acuerdo con la afirmación (DT = desviación típica)

Tabla 2. Razones de la no evaluación de las actividades de promoción de la lectura

La relación de posibles causas de la no evaluación se ampliaba con preguntas abiertas. La razón más alegada era la falta de tiempo o de recursos humanos en las bibliotecas unipersonales $(46,8 \%)$, seguida por la falta de instrumentos para medir y recoger datos $(18,7 \%)$ y, aunque parezca sorprendente, había una cierta cantidad de respuestas que manifestaban que no habían pensado en ello (9,3\%).

\section{Beneficios y factores de éxito}

El cuestionario preguntaba sobre aspectos de valoración de las actividades de promoción de la lectura por parte de las bibliotecas en términos de beneficios y eficacia. También sobre los factores que inciden en el éxito de las acciones de promoción de la lectura y los que lo dificultan.

Los profesionales consideraban que las actividades eran bien valoradas por los usuarios destinatarios, y esta valoración positiva se sumaba a los efectos beneficiosos que las prácticas de promoción podían implicar para las bibliotecas. Ante todo, las actividades ofrecen la posibilidad de crear alianzas con otros equipamientos y servicios culturales y educativos de la localidad. Otro beneficio que se le supone, 
y en el que una gran parte de los encuestados está de acuerdo, es la posibilidad de atraer nuevos públicos a la biblioteca. Curiosamente, el incremento de préstamo no se halla entre los principales beneficios a pesar de que tiene una alta consideración. Por otro lado, se detecta un rastro de escepticismo en torno a estas actividades puesto que existe un cierto grado de acuerdo en que tienen un coste elevado, generan mucho trabajo y no cumplen directamente con sus objetivos. Los resultados se presentan en la tabla 3.

\begin{tabular}{|l|c|c|}
\hline \multicolumn{1}{|c|}{ Actividades de promoción de la lectura } & Media & DT \\
\hline Son bien valoradas por los usuarios & 5,82 & 1,09 \\
\hline $\begin{array}{l}\text { Permiten crear alianzas con el entorno cultural } \\
\text { local }\end{array}$ & 5,81 & 1,15 \\
\hline Atraen nuevos públicos & 5,06 & 1,37 \\
\hline Generan más préstamos & 4,80 & 1,36 \\
\hline $\begin{array}{l}\text { Son un buen instrumento a pesar de que los } \\
\text { resultados son difíciles de medir }\end{array}$ & 4,62 & 1,48 \\
\hline Gravan excesivamente el presupuesto & 3,93 & 1,65 \\
\hline $\begin{array}{l}\text { Sirven para dar a conocer la biblioteca, pero no } \\
\text { para fomentar la lectura }\end{array}$ & 3,56 & 1,66 \\
\hline $\begin{array}{l}\text { Generan mucho trabajo para unos resultados } \\
\text { inciertos }\end{array}$ & 3,33 & 1,56 \\
\hline
\end{tabular}

Escala de valoración de 1 a 7 puntos. A mayor puntuación, más de acuerdo con la afirmación (DT = desviación típica)

Tabla 3: Grado de acuerdo con las aportaciones de las actividades de promoción de la lectura

Las actividades, en su conjunto, reportan beneficios pero ¿cuándo consideran las bibliotecas que una actividad obtiene un buen resultado? Y, ¿cuáles son las claves para el éxito?

Las bibliotecas recurren a los más variados sistemas para evaluar las actividades de promoción lectora y generan sus propias escalas de valoración de los resultados de las actividades. Hay unanimidad en considerar la satisfacción del público como el valor más aceptado. También se considera positivo que los usuarios hayan solicitado la repetición de aquella actividad o que una actividad haya atraído nuevo público. Otros indicadores que suscitan un grado de acuerdo elevado tienen que ver con los efectos generados por la actividad, como el incremento del préstamo de materiales más directamente relacionados con el tema de la misma, o el hecho de que el número de asistentes alcance o se proxime a lo esperado. Otras consideraciones, como que se hayan movilizado los documentos relacionados con la actividad o se hayan registrado desideratas en el mismo tema, no parecen suscitar tanto acuerdo. En la tabla 4 se muestran las opciones ofrecidas en esta pregunta.

Se considera que la adecuación al público al que se dirige constituye el factor principal de éxito de estas actividades; con una media similar se sitúan, por un lado, que el responsable ejecutor de la actividad tenga determinadas cualidades, como empatía, seguridad, dominio del tema, etc., que le permitan conectar y atraer al público, y por otro, el horario de la actividad. Les sigue la relación con el entorno, ya sea porque se tratan temas o autores locales o por la fama del invitado. Otros factores que parecería que deberían influir en el éxito de público como la gratuidad, la originalidad de

\begin{tabular}{|l|c|c|}
\hline \multicolumn{1}{|c|}{ Cuando... } & Media & DT \\
\hline ...el público ha salido contento & 6,29 & 0,99 \\
\hline ...el público ha solicitado repetirla & 6,25 & 0,90 \\
\hline ...ha atraído nuevo público a la biblioteca & 6,11 & 0,91 \\
\hline $\begin{array}{l}\text {...ha dinamizado el fondo más relacionado } \\
\text { con la propuesta }\end{array}$ & 5,83 & 1,04 \\
\hline $\begin{array}{l}\text {...se ha conseguido reunir el número de } \\
\text { personas esperado }\end{array}$ & 5,67 & 1,04 \\
\hline $\begin{array}{l}\text {...se han recogido desideratas relacionadas } \\
\text { con el tema }\end{array}$ & 5,06 & 1,53 \\
\hline ...la acción se ha desarrollado sin incidentes & 4,34 & 1,57 \\
\hline \begin{tabular}{l}
...se ha ajustado al presupuesto \\
\hline
\end{tabular} & 4,30 & 1,43 \\
\hline
\end{tabular}

Escala de valoración de 1 a 7 puntos. A mayor puntuación, más de acuerdo con la afirmación (DT = desviación típica)

Tabla 4. ¿Cuándo considera que una acción de promoción de la lectura ha tenido éxito?

la propuesta o que ésta invite a la participación del público, aun siendo importantes, no se consideran determinantes para el éxito. En la tabla 5 se exponen los distintos factores de éxito y su valoración por parte de los centros.

\begin{tabular}{|l|c|c|}
\hline & Media & DT \\
\hline Adecuación al público & 6,14 & 0,78 \\
\hline $\begin{array}{l}\text { Cualidades del conductor (empatía, seguridad, } \\
\text { conocimiento...) }\end{array}$ & 6,04 & 0,99 \\
\hline Horario de programación & 6,01 & 0,93 \\
\hline $\begin{array}{l}\text { Relación de la actividad con el entorno } \\
\text { (temas/autores locales, implicación de las } \\
\text { entidades...) }\end{array}$ & 5,91 & 1,05 \\
\hline Fama del conductor (mediático, local...) & 5,89 & 1,15 \\
\hline Temática (actualidad, interés) & 5,88 & 1,03 \\
\hline $\begin{array}{l}\text { Público a quien se dirige (infantil, jóvenes, } \\
\text { adultos) }\end{array}$ & 5,86 & 1,07 \\
\hline Medio de difusión que se utiliza & 5,78 & 1,07 \\
\hline Gratuidad & 5,76 & 1,29 \\
\hline Implicación de entitades locales & 5,34 & 1,49 \\
\hline Adecuación del espacio donde se realiza & 5,34 & 1,18 \\
\hline $\begin{array}{l}\text { Grado de implicación por parte del lector } \\
\text { (comentario de lecturas, participación en } \\
\text { talleres...) }\end{array}$ & 5,31 & 1,18 \\
\hline Variedad & 5,10 & 1,21 \\
\hline Originalidad & 4,93 & 1,24 \\
\hline
\end{tabular}

Escala de valoración de 1 a 7 puntos. A mayor puntuación, más de acuerdo con la afirmación (DT = desviación típica)

Tabla 5. Grado en que estos factores parecen determinantes para el éxito de una actividad

\section{¿Qué es lo que de verdad funciona? Larga vida a los clubs de lectura}

Como información complementaria, se preguntaba a los encuestados qué tipo de propuesta o de formato de actividad consideraban más eficaz para la promoción de la lectura en las bibliotecas. Las 25 posibilidades planteadas reciben todas una puntuación por encima del valor medio de la escala, y con una DT no superior a 1,5, lo que indica que los profesionales consideran que la promoción lectora requiere una gran variedad de propuestas dirigidas a los distintos 
públicos y que, en alguna medida, su eficacia deriva de la complementariedad. Aun así destaca la unanimidad en considerar a los clubs de lectura (para adultos, pero también los destinados a jóvenes y niños) los formatos más idóneos para la promoción de la lectura. A continuación, pero con una cierta diferencia, se sitúan los encuentros con autores y las actividades de narraciones de cuentos para niños (no así las narraciones para adultos, consideradas mucho menos eficaces). Se citan otras acciones cuya eficacia se considera menor: los concursos literarios, el bookcrossing o práctica de dejar libros en lugares públicos -en este caso las bibliotecas- para que los recojan otros lectores, las actividades relacionadas con proyecciones cinematográficas (cine forum) y los talleres no específicamente relacionados con lectura y escritura. En estos casos, existen más de dos puntos de diferencia con la actividad mejor valorada, como puede verse en la tabla 6.

\section{Conclusión}

Las bibliotecas tienen una gran confianza en los beneficios que pueden generar las actividades de promoción a la lectura aunque en muy pocos casos miden su impacto. El hecho de no disponer de instrumentos y mecanismos para recabar indicadores que les permitan evaluar de manera más o me-

\begin{tabular}{|c|c|c|}
\hline & Media & DT \\
\hline Clubs de lectura para adultos & 6,30 & 0,91 \\
\hline Encuentros con autores & 5,87 & 0,98 \\
\hline Clubs de lectura para jóvenes & 5,82 & 1,38 \\
\hline Narraciones de cuentos para niños & 5,76 & 1,15 \\
\hline Clubs de lectura para niños & 5,75 & 1,43 \\
\hline Exposición de novedades & 5,73 & 1,04 \\
\hline Intercambio de opiniones con el bibliotecario & 5,65 & 1,18 \\
\hline Servicios de alerta de novedades & 5,49 & 1,13 \\
\hline Visitas infantiles y escolares & 5,47 & 1,19 \\
\hline Presentaciones de libros & 5,22 & 1,27 \\
\hline Exposiciones temáticas de libros & 4,98 & 1,40 \\
\hline $\begin{array}{l}\text { Opiniones y recomendaciones por escrito de } \\
\text { los lectores }\end{array}$ & 4,98 & 1,21 \\
\hline $\begin{array}{l}\text { Blog con valoraciones y opiniones de los } \\
\text { lectores }\end{array}$ & 4,92 & 1,33 \\
\hline Conferencias, charlas y mesas redondas & 4,85 & 1,26 \\
\hline Rutas literarias & 4,85 & 1,23 \\
\hline Guías de lectura & 4,75 & 1,25 \\
\hline $\begin{array}{l}\text { Espectáculos (representaciones teatrales, } \\
\text { marionetas...) }\end{array}$ & 4,71 & 1,50 \\
\hline Talleres de escritura & 4,66 & 1,35 \\
\hline Lecturas en voz alta por parte de los lectores & 4,65 & 1,39 \\
\hline Narraciones orales para adultos & 4,60 & 1,32 \\
\hline Actos relacionados con las exposiciones & 4,44 & 1,29 \\
\hline Concursos literarios & 4,34 & 1,45 \\
\hline Bookcrossing & 4,30 & 1,47 \\
\hline Cine forum & 4,26 & 1,39 \\
\hline Talleres diversos & 4,16 & 1,31 \\
\hline
\end{tabular}

Escala de valoración de 1 a 7 puntos. A mayor puntuación, más de acuerdo con la afirmación (DT = desviación típica)

Tabla 6. ¿En qué grado estas acciones fomentan la lectura? nos objetiva las acciones de promoción de la lectura no es un freno a estas prácticas.

Por otro lado, no parece que los métodos de evaluación de la práctica lectora incorporen elementos de mayor calado como la frecuencia de la lectura, la comprensión lectora, el desarrollo de la capacidad crítica, la autonomía en la selección, etc. limitándose al cómputo cuantitativo (número de público asistente, número de libros prestados) y a las encuestas de satisfacción.

Aunque la bibliografía profesional ya advierte de la dificultad de llevar a cabo estos procesos de evaluación, es innegable que se debería poder ensayar un protocolo que, al menos, determinara hasta qué punto las acciones emprendidas por la biblioteca tienen efectos (aunque mínimos o difícilmente cuantificables) para una mejora de la práctica lectora y cuáles son simplemente acciones para acercar la biblioteca a sus usuarios. Evidentemente, la mejora será a largo plazo y esperamos que los estudios que pretendan cuantificar la evaluación de estas acciones evidencien estos cambios, algo que tras más de treinta años de promoción lectora no ha sucedido.

\section{Bibliografía}

Baró, Mónica; Casas-Poves, Juana; Mañà, Teresa; ReyesCamps, Lourdes. "La promoció de la lectura a les biblioteques públiques de Catalunya: un terreny pròsper que cal endreçar". En: 12 Jornades catalanes d'informació i documentació, 2010.

http://www.cobdc.org/jornades/12JCD/materials/comuni cacions/REYES_promocio_lectura_BBPP.pdf

Baró, Mónica; Mañà, Teresa. Estudi sobre les accions de foment de la lectura a les biblioteques públiques de Catalunya. Barcelona: DG de Coop. Cultural, Generalitat de Catalunya, 2009.

http://www20.gencat.cat/docs/Biblioteques/Tematic/Do cuments/Arxiu/Activitats/Estudi_foment_lectura.pdf

Farmer, Lesley; Stricevic, Ivanka. El uso de la investigación para promover la alfabetización y la lectura en las bibliotecas: guía para los bibliotecarios. La Haya: IFLA, 2011.

http://www.ifla.org/files/hq/publications/professional-re port/126.pdf

Greenwood, Helen; Davies J. Eric. "Designing tools to fill the void: a case study in developing evaluation for reading promotion projects". Performance measurement and metrics, 2004 , v. 5, n. 3, pp. 106-111.

http://dx.doi.org/10.1108/14678040410570120

Train, Briony; Elkin, Judith. "Measuring the unmeasurable: reader development and its impact on performance measurement in the public library sector". Library review, 2001, v. 50, n. 6, pp. 295-304. http://dx.doi.org/10.1108/EUM0000000005598

Wilson, Kerry; Train, Briony. Reader development evaluation framework. Sheffield: The University of Sheffield, The British Council, 2004.

http://www.shef.ac.uk/content/1/c6/07/01/24/CPLIS\%20\%20British\%20Council\%20RD\%20Evaluation\%20Framewor k.pdf 


\section{MiBjb]jotece}

Actualidad bibliotecaria

Fotografía momentos de lectura

Opinión

Recomendaciones de lectura Escolares

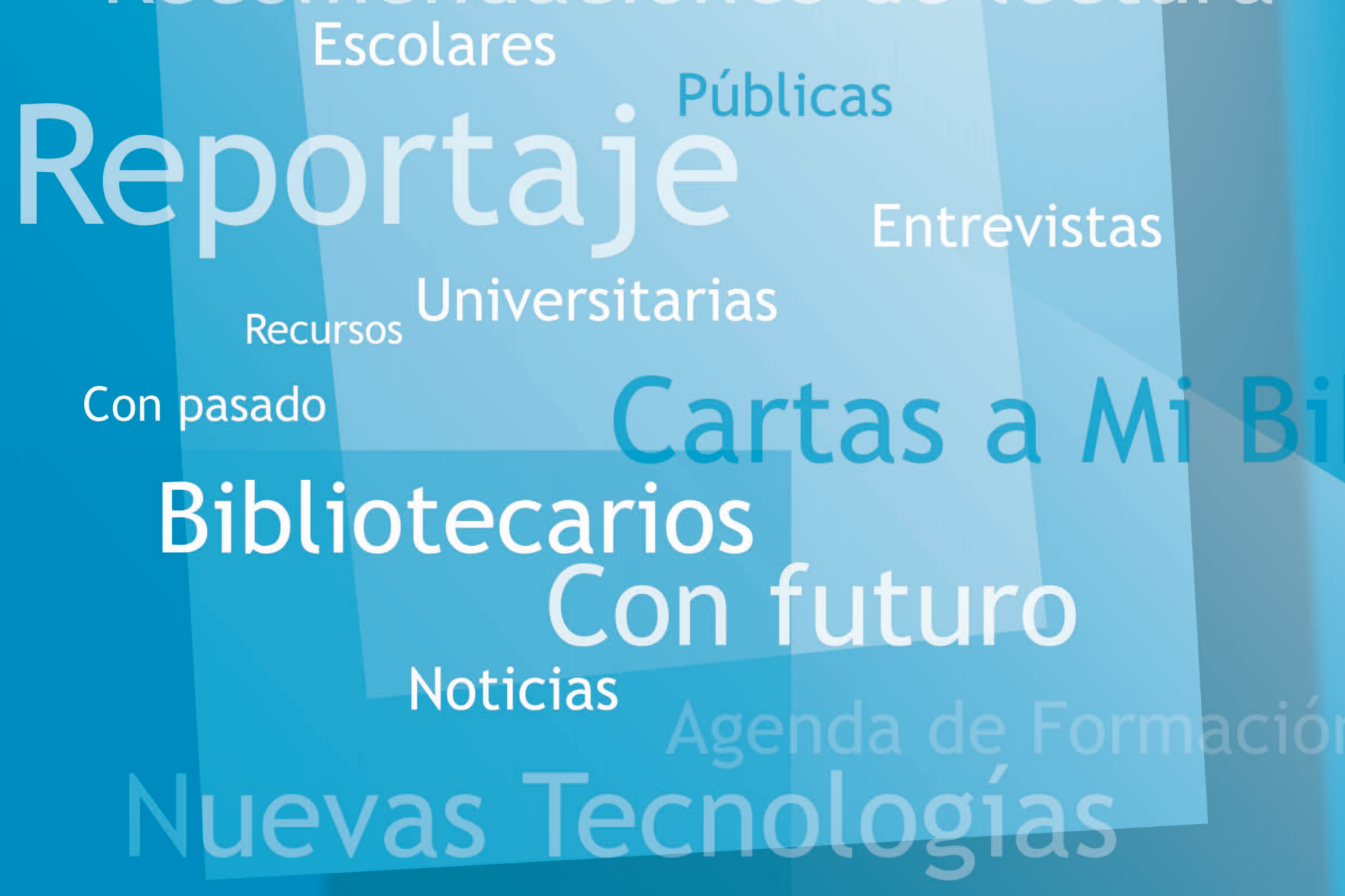

Gestión documental

www.mibiblioteca.org 